\title{
Análise da eficiência de modelos de decaimento de primeira ordem na previsão da emissão de gás de efeito estufa em aterros sanitários brasileiros
}

\author{
Analysis of the efficiency of first-order decay models in \\ forecasting greenhouse gas emission in Brazilian sanitary landfills
}

Mauro Meirelles Santos', Celso Romanel${ }^{2}$, Ana Ghislane Henriques Pereira van Elk ${ }^{3}$

口-

\begin{abstract}
RESUMO
O objetivo deste artigo foi avaliar a eficiência de modelos de primeira ordem para previsão da geração de biogás em aterros sanitários, comparando resultados previstos com aqueles disponíveis em relatórios de monitoramento de captação ao longo de anos de operação. Os aterros sanitários estudados neste trabalho operam no âmbito de projetos de Mecanismo de Desenvolvimento Limpo (MDL), que podem receber incentivos financeiros - certificados de redução de emissões ou créditos de carbono. Foram selecionados quatro aterros sanitários brasileiros (Nova Iguaçu, Paulínia, Caieiras e Bandeirantes) que dispunham das quantidades anuais de resíduos sólidos efetivamente depositadas durante o primeiro período de crédito de seteanos, eliminando assimuma importantefonte de incertezas do modelo de previsão. Limitações dos modelos recomendados pelas diretrizes do Intergovernmental Panel on Climate Change (IPCC) são discutidas, incluindo erros intrínsecos na formulação matemática e de má interpretação no tempo para início da geração do biogás. É também proposta uma modificação na ferramenta de projeto CDM Tool atualmente utilizada em projetos MDL de emissão de biogás em depósitos de resíduos sólidos.
\end{abstract}

Palavras-chave: aterros sanitários; emissão de biogás; mecanismo de desenvolvimento limpo; captação de metano; modelos de primeira ordem.

\begin{abstract}
The objective of this study was to evaluate the efficiency of first-order models for prediction of biogas emission in landfills, comparing forecasts with observations registered along the years of landfill operation. The landfills analyzed in this research operate within Clean Development Mechanism (CDM) that may receive financial incentives - certified emission reductions or carbon credits. Four Brazilian landfills (Nova Iguaçu, Paulinia, Caieiras and Bandeirantes) have been selected, for which the annual quantities of waste actually deposited during the first seven-year period of carbon credits were available, thus eliminating a major source of uncertainty in the forecasting models. Limitations of the models of the first order recommended by the guidelines of the Intergovernmental Panel on Climate Change (IPCC) are discussed, including intrinsic errors in the mathematical formulation and misinterpretation of the starting time for prediction of biogas emission. It is also proposed a modification in the CDM Tool model, currently used for biogas emission in solid waste deposits operating as CDM projects.
\end{abstract}

Keywords: landfills; biogas emission; clean development mechanism; methane capture; first order forecasting models.

\section{INTRODUÇÃO}

Com a criação do mercado de carbono regulado pelo Mecanismo de Desenvolvimento Limpo (MDL), surgiram no Brasil vários empreendimentos de aterros sanitários com captação de biogás. Desde 2005, quando o Protocolo de Quioto entrou em vigor, até outubro de 2016, 379 projetos brasileiros foram registrados no Conselho Executivo do

MDL. O Protocolo de Quioto, prolongado de 2013 a 2020 pela Emenda de Doha, foi suplantado pelo histórico Acordo de Paris, compromisso internacional para reduzir emissões de gases de efeito estufa na atmosfera, aprovado por 195 países na $21 .{ }^{a}$ Conferência das Partes (COP21) da Convenção-Quadro das Nações Unidas sobre Mudanças Climáticas (UNFCCC). Por esse acordo, em vigor desde novembro de 2016, o

\footnotetext{
'Mestre em Engenharia Urbana e Ambiental pela Pontifícia Universidade Católica do Rio de Janeiro (PUC-Rio). Doutorando do Programa de Planejamento Energético da COPPE, Universidade Federal do Rio de Janeiro (UFRJ) - Rio de Janeiro (RJ), Brasil.

2Ph.D. pela Universidade do Arizona, EUA. Professor do Departamento de Engenharia Civil da PUC-Rio - Rio de Janeiro (RJ), Brasil.

${ }^{3}$ D.Sc. pela Universidade de Oviedo, Espanha. Professora do Departamento de Engenharia Sanitária e do Meio Ambiente da Universidade do Estado do Rio de Janeiro (UERJ) Rio de Janeiro (RJ), Brasil

Endereço para correspondência: Ana Ghislane Henriques Pereira van Elk - Rua São Francisco Xavier, 524, Pavilhão João Lyra Filho, $5^{\circ}$ andar, sala 5029, Bloco F Maracanã 24550-400 - Rio de Janeiro (RJ), Brasil - E-mail: anavanelk@gmail.com

Recebido: 01/O2/16 - Aceito: 24/10/16 - Reg. ABES: 156311
} 
governo brasileiro compromete-se a cortar as emissões do país em $37 \%$ até 2025 e em 43\% até 2030, tendo como referência o ano de 2005.

O projeto e a operação de aterros para extração e utilização de biogás requerem previsões de emissão para garantir a viabilidade econômica do empreendimento e sua conformidade ambiental. Nos primeiros projetos MDL no Brasil, ocorreu significativa superestimação da quantidade de biogás gerada nos aterros sanitários, uma vez que não foram adequadamente considerados vários fatores que influenciam na quantidade e qualidade do biogás, como a composição e idade dos resíduos, o projeto e a forma de operação do aterro e o clima da região. Adicionalmente, não foram apropriadamente contabilizadas as perdas por emissões fugitivas, por difusão e/ou advecção, nem por processos de oxidação.

A estimativa da quantidade de gás gerada no aterro pode ser feita com auxílio de modelos de previsão de ordem zero, primeira ordem ou modelos multifásicos. A maioria dos projetos aplica modelos de primeira ordem, que assumem emissão de metano exponencialmente decrescente com o tempo, como por exemplo: LandGEM (USEPA, 2005), Scholl-Canyon (WORLD BANK, 2004) e Intergovernmental Panel on Climate Change (IPCC), em suas várias versões (IPCC 1997; 2000; 2006).

Thompson et al. (2009) compararam a quantidade de metano captada em 35 aterros sanitários no Canadá, concluindo que os modelos Scholl-Canyon e LandGEM apresentaram erros inferiores a 100\%, com subestimativas de produção no modelo LandGEM. Vários outros autores realizaram investigações semelhantes, como Barlaz et al. (2004), Spokas et al. (2006), Terraza, Willumsen e Guimarães (2007), Viana (2011) e Amini, Reinhardt e Niskanen (2013).

O objetivo deste trabalho foi analisar a eficiência dos modelos IPCC de primeira ordem, utilizados em inventários nacionais e em estimativas de emissões de metano em aterros sanitários, e do modelo CDM Tool, ferramenta de cálculo de emissões em aterros sanitários no âmbito de projetos MDL. Foram comparadas as previsões de emissão e a efetiva captação de biogás em quatro aterros brasileiros - Central de Tratamento de Resíduos Sólidos (CTR) Nova Iguaçu, Bandeirantes, Caieiras e ESTRE Paulínia - registrados no MDL e que dispõem de relatórios de monitoramento referentes ao primeiro período de créditos de sete anos. Na análise comparativa da capacidade de previsão dos modelos, a eficiência foi estabelecida em relação às quantidades de metano efetivamente captadas, de acordo com informações disponíveis nos relatórios de monitoramento dos aterros. $\mathrm{O}$ trabalho também discutiu erros de interpretação dos modelos IPCC e propôs a aplicação de uma correção na ferramenta CDM Tool.

\section{Modelos Intergovernmental Panel on Climate Change}

Modelos de decaimento de primeira ordem consideram a variação anual das emissões de metano com base em dois parâmetros: a constante de decaimento $k$ (Equação 1) e o potencial de geração de metano $L_{0}$.

$k=\ln (2) / t^{1 / 2} \quad(\text { ano })^{-1}$

Em que:

$t^{1 / 2}$ é a meia-vida (em anos) do carbono degradável no aterro.

O valor de k define o intervalo de tempo de geração de metano a partir da deposição dos resíduos, o qual pode ser afetado pelo teor de umidade do aterro, temperatura, potencial de oxidação, fator $\mathrm{pH}$, densidade e granulometria dos resíduos.

O potencial de geração de metano $L_{0}\left(t \mathrm{CH}_{4} / \mathrm{t}\right.$ resíduos ou $\mathrm{m}^{3} \mathrm{CH}_{4} / \mathrm{t}$ resíduos) representa a quantidade desse gás produzida pela massa de resíduos. Esse valor é o máximo numa situação em que os resíduos sólidos estão em condição anaeróbia, e o metano é emitido ao longo dos anos com decaimento exponencial no tempo. O valor de $L_{0}$ é sensível a alterações na composição dos resíduos, a qual pode variar como resultado de mudanças de padrão de consumo da comunidade ou expansão de programas de minimização de resíduos. Para que $L_{0}$ represente o máximo de emissão por tonelada de resíduos com massa $\mathrm{M}$, a emissão de metano Q como função do tempo t é expressa pela Equação 2:

$Q(\mathrm{t})=k \times M \times L_{0} \times e^{-k t}$

Modelo Intergovernmental Panel on Climate Change (1997) O modelo IPCC (1997) estima a captação de $\mathrm{CH}_{4}$ por meio de resíduos depositados no aterro em um único ano, conforme Equação 3.

$Q_{T, x}=k \times R_{x} \times L_{0} \times e^{-k(T-x)}$

Em que:

$Q_{T, x}$ é a quantidade de metano emitida no ano corrente $T$ pelos resíduos $R_{x}(\mathrm{Mg})$ depositados no ano $x$.

Se na aplicação da Equação 3 a primeira emissão, correspondente ao ano $T=1$, for calculada a partir dos resíduos colocados no primeiro ano $(x=1)$, então obtém-se a Equação 4:

$Q_{1,1}=k \times R_{1} \times L_{0} \times e^{-k(1-1)}=k \times R_{1} \times L_{0}$

Por outro lado, se a primeira emissão $(T=1)$ for calculada a partir dos resíduos colocados no ano anterior $(x=0)$, então obtém-se a Equação 5:

$Q_{1,0}=k \times R_{0} \times L_{0} \times e^{-k(1-0)}=k \times R_{0} \times L_{0} \times e^{-k}$ 
Considerando o valor exato obtido pela integração do balanço de massa, a quantidade de metano é superestimada pela Equação 4 e subestimada pela Equação 5. Ambas utilizam a regra dos retângulos para aproximação do valor da integral (Figura 1). Na Equação 4 o cálculo da emissão no ano $T$ com base nos resíduos lançados no próprio ano $x=T$ gera superestimação, porque inclui as áreas escuras compreendidas entre a curva contínua e os retângulos do esquema de quadratura na Figura 1A, enquanto a determinação da emissão no ano $T$, tendo em vista os resíduos no ano anterior $x=T$-1, produz subestimação por excluir as áreas escuras da Figura 1B.

O cálculo da emissão pela Equação 4, admitindo o início da emissão no próprio ano da disposição dos resíduos, foi a interpretação adotada nos primeiros projetos de MDL que tiveram como base as recomendações das diretrizes (IPCC,1997).

\section{Modelo Intergovernmental Panel on Climate Change (2000)}

O modelo de decaimento de primeira ordem recomendado pelas diretrizes IPCC (2000) é expresso pela Equação 6 como:

$Q_{t, x}=\sum_{x}^{t} A \times k \times M S W_{T}(\mathrm{x}) \times M S W_{F}(\mathrm{x}) \times L_{0}(\mathrm{x}) \times e^{-k(t-x)}$

Em que:

$Q_{t, x}$ : quantidade de $\mathrm{CH}_{4}$ emitida no ano $t\left(\mathrm{Gg}_{\text {ano }}{ }^{-1}\right)$;

$t$ : ano do inventário;

$x$ : ano de deposição dos resíduos;

$k$ : constante de decaimento;

$A=\left(1-\mathrm{e}^{-\mathrm{k}}\right) / k$ : fator de correção do valor da quadratura numérica; $M S W_{\mathrm{T}}(x)$ : total de resíduos sólidos depositados até o ano $x$ ( $\left.\mathrm{Gg} / \mathrm{ano}\right)$; $M S W_{\mathrm{F}}(x)$ : fração dos resíduos sólidos depositados no ano $x$;
$L_{0}(x)$ : potencial de geração de metano equivalente ao produto $[M C F(x)$ $\times D O C(x) \times D O C_{\mathrm{F}} \times F \times 16 / 12\left(\mathrm{Gg} \mathrm{CH}_{4} / \mathrm{Gg}\right.$ resíduos $\left.)\right]$;

$M C F(x)$ : fator de correção do metano no ano $x$ (fração);

$\operatorname{DOC}(x)$ : carbono orgânico degradável (COD) no ano $x$ (fração)

(Gg C/Gg resíduos);

$D O C_{\mathrm{F}}$ : fração do COD que se decompõe;

F: fração em volume de $\mathrm{CH}_{4}$ no gás de aterro; e

16/12: fator de conversão de carbono (C) para metano $\mathrm{CH}_{4}$.

A emissão de metano a partir de resíduos depositados no aterro no ano $1(x=1)$, por exemplo, pode ser calculada no mesmo ano $(t=1)$. Nesse caso, a previsão da quantidade de metano é calculada pela Equação 7:

$Q_{1,1}=A \times k \times R_{1} \times L_{0}(1)$

Aqui, o produto $M S W_{\mathrm{T}}(x) \times M S W_{\mathrm{F}}(x)$ da Equação 6 foi substituído por $R_{x}$, que tem o mesmo significado que o termo correspondente da Equação 3.

Comparando as Equações 7 (IPCC, 2000) e 3 (IPCC, 1997), verifica-se que a quantidade anual de metano prevista difere do fator de correção $A$ introduzido na Equação 7. Não há nenhuma indicação nas diretrizes IPCC (2000) sobre como esse fator foi obtido, mas Cassini, Breda e Nascimento (2013) demonstraram que $A=\left(1-e^{-k}\right) / k$.

Como a Equação 6 é integrada do ano $t$ para o ano $t+1$, não é possível contabilizar a emissão de metano no primeiro ano com o modelo IPCC (2000), como ilustrado na Figura 2A. As diretrizes IPCC (2006) interpretam que as emissões não devem ser calculadas no próprio ano da deposição dos resíduos, ao contrário da prática corrente observada em muitos inventários nacionais e em atividades de projeto MDL que utilizaram as diretrizes IPCC $(1997 ; 2000)$. Assim, para a estimativa mais
A

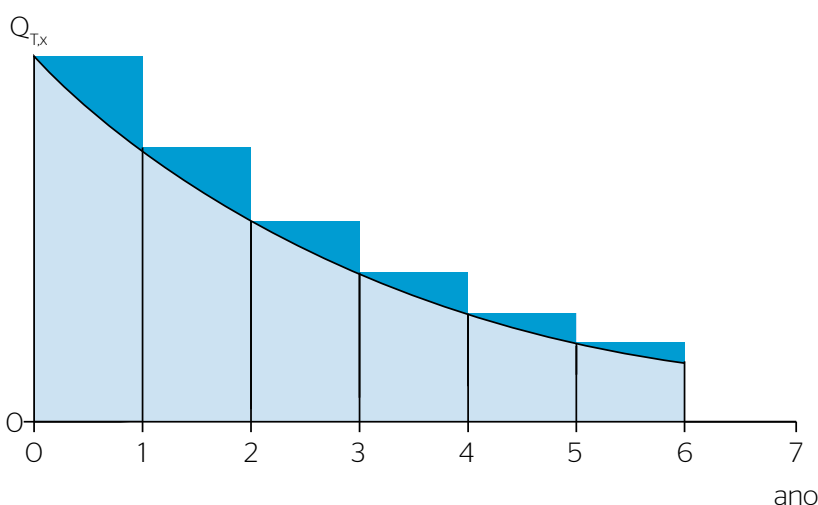

B

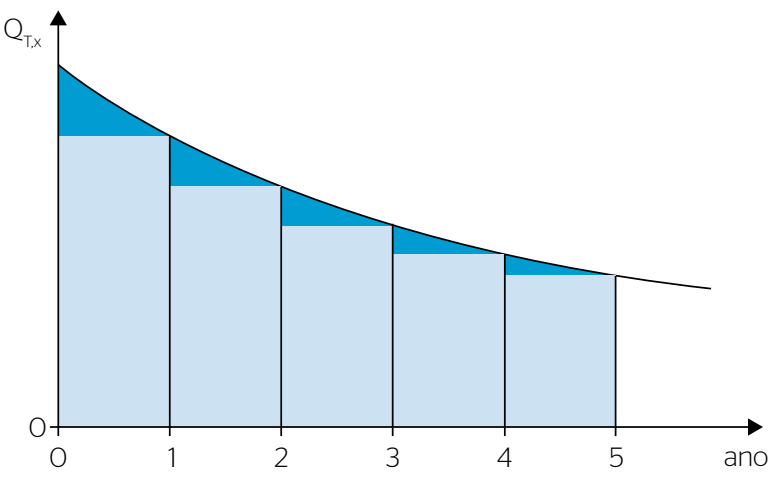

Figura 1 - (A) Áreas escuras indicam quantidade aproximada de metano $Q_{T x}$ superestimada pela Equação 4; (B) áreas escuras indicam quantidade aproximada de metano $Q_{T, x}$ subestimada pela Equação 5 . 
adequada da quantidade de metano calculada pela Equação 6, recomenda-se substituir o fator de correção $A$ por um novo fator de correção $A$ ', considerando a integração do ano $(t-1)$ para o ano $t$, conforme Equação 8:

$A^{\prime}=\left(e^{k}-1\right) / k$

Tem-se então o entendimento implícito de que as emissões são sempre calculadas a partir do ano seguinte ao ano da deposição dos resíduos no aterro. Em outras palavras, a quantidade de resíduos depositada durante o primeiro ano pode ser considerada totalmente colocada no aterro no meio do primeiro ano. Existe, portanto, um atraso típico de seis meses (IPCC, 2006) não apresentado nas diretrizes anteriores (IPCC 1997; 2000) para o início do processo de digestão anaeróbia, o que significa que o metano começa a ser gerado apenas no início do segundo ano. Essa interpretação implica duas consequências na utilização do modelo IPCC (2000) com o novo fator de correção $A^{\prime}$ : deslocamento no tempo de um ano na curva exponencial das emissões; emissões que ocorrem durante $\mathrm{o}$ ano $t$ devem ser calculadas pela integral definida entre os anos $(t-1)$ e $t$. Na Figura 2B, observa-se que, no primeiro ano, não há contabilização de emissão, que somente ocorre no segundo ano, entre $t=1$ e $t=2$.

\section{Modelo Intergovernmental Panel on Climate Change (2006)}

Nas diretrizes IPCC (2006) o modelo de decaimento para estimativa das emissões de metano é baseado em duas equações principais (Equações 9 e 10):

$$
\begin{aligned}
& D^{D O O C_{m a T}}=D D O C_{m d T}+D D O C_{m d T-1} \times e^{-k} \\
& D D O C_{m \text { decompT }}=D D O C_{m a T-1} \times\left(1-e^{-k}\right)
\end{aligned}
$$

Em que:

$T$ : ano do inventário;

$D D O C_{\text {mat }}$ : massa de COD acumulada ao final do ano $T(\mathrm{Gg})$;

$D D O C_{\mathrm{mdT}}$ : massa de COD depositada no ano $T(\mathrm{Gg})$;

$D D O C_{\text {mdT-1 }}$ : massa de COD depositada no ano $T-1(\mathrm{Gg})$;

k: constante de decaimento;

$D D O C_{\mathrm{m} \text { decomp }}$ : massa de COD decomposta no ano $T(\mathrm{Gg})$; e

$D D O C_{\text {maT-1 }}$ : massa de COD acumulada ao final do ano $T-1(\mathrm{Gg})$.

As diretrizes também oferecem várias opções para valores da constante de decaimento $k$ e de outros parâmetros que fazem parte do cálculo da massa de carbono degradável no processo anaeróbio, considerando cada tipo de fração de resíduo. Essas diretrizes admitem atrasos na decomposição, normalmente de seis meses.

\section{Ferramentas para projetos de Mecanismo de Desenvolvimento Limpo em aterros sanitários}

\section{Modelo CDM Tool}

As atividades de projeto envolvendo emissão de biogás que buscam registro no âmbito do MDL devem usar a norma Methodological Tool: Emissions from Solid Waste Disposal Sites - SWDS (UNFCCC, 2011), ou simplesmente a CDM Tool, para a avaliação da quantidade de metano, expressa em equivalentes de dióxido de carbono $\left(\mathrm{CO}_{2}\right)$ pela Equação 11:

$B E_{C H 4, S W D S, y}=\varphi_{y} \times\left(1-f_{y}\right) \times G W P_{G H 4} \times(1-O X) \times \frac{16}{12} \times F \times$

$D O C_{f, y} \times M C F_{y} \times \sum_{x=1}^{y} \sum_{j} W_{j, x} \times D O C_{j} \times e^{-k_{j}(y-x)} \times\left(1-e^{-k j}\right)$
A

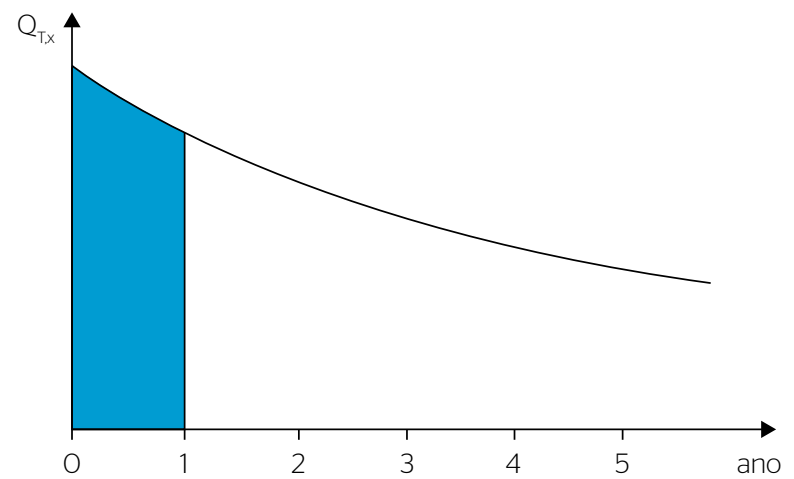

B

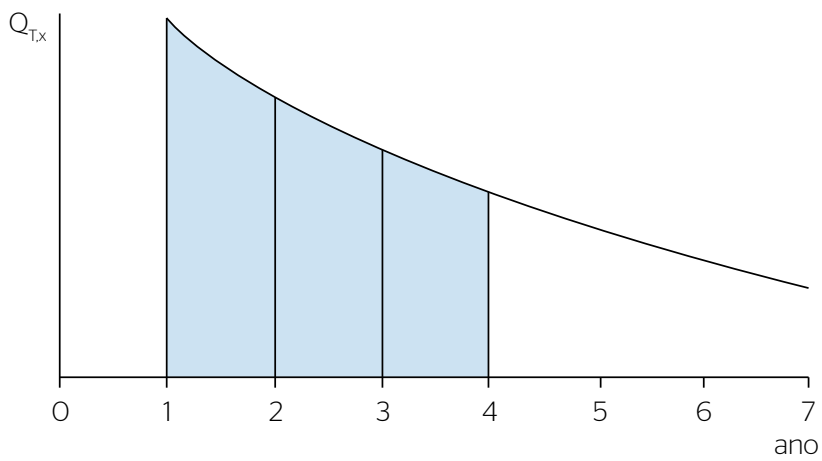

Figura 2 - (A) A área escura representa emissão não contabilizada no primeiro ano (IPCC, 2000); (B) utilização do modelo IPCC (2000) com novo fator de correção $A^{\prime}$ e desconsideração de emissão no primeiro ano da deposição de resíduos. 
Em que:

$B E_{\mathrm{CH} 4, \mathrm{SWDS}, \mathrm{y}}$ : emissão de metano da linha de base que ocorre no ano $y$ considerando a deposição de resíduos sólidos durante o período em que termina no ano $y$ ( $\left.\mathrm{t} \mathrm{CO}_{2 \mathrm{e}} / \mathrm{ano}\right)$;

$\varphi_{\mathrm{y}}$ : fator de correção que leva em conta as incertezas do modelo no ano $y$; $f y$ : fração de metano capturada e queimada, oxidada ou utilizada de outra maneira a fim de evitar as emissões de metano para a atmosfera no ano $y$; $G W P_{\mathrm{CH} 4}$ : potencial de aquecimento global do metano;

$O X$ : fator de oxidação que reflete a quantidade de metano oxidada no solo ou em outro material que recubra o resíduo;

F: fração de metano no biogás (fração de volume);

$D O C_{f, y}$ : fração de COD que se decompõe nas condições específicas do aterro para o ano $y$ (fração de peso);

$M C F_{\mathrm{y}}$ : fator de correção do metano no ano $y$ (valor igual a 1 para aterros sanitários);

$x$ : número de anos em que os resíduos estão depositados, estendendo-se do primeiro ano $(x=1)$ para o ano $y(x=y)$, sendo $y$ um período consecutivo de 12 meses;

$W_{\mathrm{j}, \mathrm{x}}$ : quantidade de resíduos sólidos do tipo $j$ depositados no ano $x$; $D O C_{j}$ : fração de COD no resíduo de tipo $j$ (fração de peso); e $k_{j}$ : constante de decaimento para o resíduo tipo $j\left(\mathrm{ano}^{-1}\right)$.

Nesse ponto a Equação 11 pode ser simplificada com base nas seguintes premissas: apenas geração de metano, com a remoção do termo $G W P_{\mathrm{CH} 4}$; nenhum metano é capturado nem queimado $\left(f_{\mathrm{y}}=0\right)$; não há oxidação pela superfície $(O X=0)$; apenas uma única fração $j$ de resíduo contribui para a emissão no ano $y$; fator de incerteza $\left(\varphi_{\mathrm{y}}=1\right)$. Logo, obtém-se a Equação 12:

$B E_{y}=\left(1-e^{-k}\right) \times W_{x} \times L_{0} \times e^{-k(y-x)}=\left(\frac{1-\mathrm{e}^{-\mathrm{k}}}{\mathrm{k}}\right) \times k \times W_{x} \times L_{0} \times e^{-k(y-x)}$

$L_{0}$ é explicada pela Equação 13:

$L_{0}=\frac{16}{12} \times F \times D O C_{f, y} \times D O C_{j}$

Considerando o fator de correção $A=\left(1-e^{-k}\right) / k$ observa-se que a Equação 12 é equivalente à Equação 6 (IPCC, 2000). Logo, pode-se concluir que o mesmo erro intrínseco associado ao modelo IPCC (2000), incluindo a incorreta interpretação que estima as emissões a partir do ano da deposição, também está presente nas previsões efetuadas com o modelo CDM Tool. Uma correção similar àquela sugerida nas diretrizes IPCC (2006), com o novo fator de correção $A^{\prime}$ da Equação 8, é sugerida em uma modificação do modelo CDM Tool, conforme descrito a seguir.

\section{Modelo CDM Tool Modificado}

O modelo CDM Tool Modificado foi proposto por Santos (2014) com a substituição, na Equação 11, do termo $\left(1-e^{-k j}\right)$, relacionado com o fator de correção $A=\left(1-\mathrm{e}^{-\mathrm{k}}\right) / k$ da Equação 6, pelo termo ( $\left.e^{\mathrm{kj}}-1\right)$, associado com o novo fator de correção $A^{\prime}=\left(\mathrm{e}^{\mathrm{k}}-1\right) / k$ da Equação 8.

O modelo CDM Tool Modificado é representado pela Equação 14, incorporando também a interpretação de que a emissão deve ser calculada a partir do ano seguinte à deposição dos resíduos:

$B E_{C H 4, S W D S, y}=\varphi_{y} \times\left(1-f_{y}\right) \times G W P_{G H 4} \times(1-O X) \times \frac{16}{12} \times F \times$

$D O C_{f, y} \times M C F_{y} \times \sum_{x=1}^{y-1} \sum_{j} W_{j, x} \times D O C_{j} \times e^{-k_{j}(y-x)} \times\left(e^{k j}-1\right)$

Em que:

$y$ é o ano do período de crédito no qual as emissões de metano são calculadas, com a restrição $y \geq x+1$ por conta do atraso de seis meses para o início do processo de decomposição.

\section{Aterros sanitários brasileiros analisados}

De acordo com procedimentos do MDL, aterros sanitários devem ter seus modelos de previsão de geração de biogás e respectivos parâmetros reavaliados por ocasião da renovação do projeto ao final de um período de créditos de sete anos. Quatro projetos no Brasil enquadram-se nessa condição: CTR Nova Iguaçu (antigo NovaGerar), Bandeirantes, Caieiras e ESTRE Paulínia.

$\mathrm{Na}$ análise comparativa entre os modelos de previsão de captação de metano utilizados em cada aterro, foram tomados os seguintes cuidados: todos os cálculos foram refeitos, a fim de verificar os valores de emissão apresentados no respectivo Project Design Document (PDD), utilizando os parâmetros originalmente nele indicados - nos primeiros PDDs não havia planilha disponível, exceto para o aterro ESTRE Paulínia; já nos segundos PDDs, todas as planilhas estavam publicadas no site do MDL —; para atingir os resultados apresentados nos respectivos PDDs, algumas correções nos parâmetros dos modelos foram necessárias, com base em estudos de retroanálise; como os segundos PDDs forneceram as quantidades de resíduos depositadas nos sete anos anteriores, os valores atualizados das quantidades de resíduos foram considerados em todos os modelos de previsão de geração de metano. As diferenças anuais das quantidades de resíduos entre os primeiros PDDs (que fizeram previsão) e os segundos PDDs (que informaram o histórico do aterro) foram significativas em três dos projetos. Em Nova Iguaçu o aumento médio da quantidade de resíduos foi de $72 \%$ (mínimo de 24\%, máximo de 168\%), em ESTRE Paulínia houve aumento médio de 106\% (mínimo de 22\%, máximo de 180\%), e em Caieiras foi observado aumento médio de $66 \%$ (mínimo de 3\%, máximo de 136\%).

\section{CTR Nova Iguaçu: projeto de aproveitamento de biogás}

O projeto CTR Nova Iguaçu, anteriormente conhecido como NovaGerar, situa-se em Nova Iguaçu, na região metropolitana da cidade do Rio de Janeiro. Possui dois locais de deposição de resíduos: Marambaia, um lixão 
que operou como aterro controlado de 1988 a 2002, e o aterro sanitário de Nova Iguaçu, que começou a operar em 2003. O primeiro período de créditos foi de $1 .^{\circ}$ de julho de 2004 a 30 de junho de 2011, mas o projeto tornou-se operacional apenas em 15 de março de 2007. O segundo período de créditos, de novembro de 2011, considerou somente o aterro sanitário de Nova Iguaçu, com os correspondentes relatórios de monitoramento (NOVA IGUAÇU, 2014), contendo dados até 31 de dezembro de 2012.

Como há dois aterros envolvidos e o de Marambaia possui dados completos das séries anuais de deposição de resíduos, o potencial de geração de metano foi determinado para esse aterro, e o mesmo valor foi assumido para o aterro de Nova Iguaçu. O primeiro PDD utilizou o modelo IPCC (1997), com o máximo de emissão a partir do ano da deposição, constante de decaimento $k=0,1$ ano $^{-1}$ e potencial de geração $L_{0}=117,14 \mathrm{~m}^{3} \mathrm{CH}_{4} \cdot \mathrm{t}^{-1}$ resíduos.

No presente trabalho, o potencial de geração de metano foi recalculado, com base nos resultados apresentados para cada ano no PDD e determinados os parâmetros que os geraram, visto que os valores indicados originalmente não são coerentes com a previsão de extração de metano apresentada. Na retroanálise, levou-se em conta $85 \%$ de eficiência de coleta e $20 \%$ de desconto na linha de base. Para o segundo PDD foi empregado o modelo CDM Tool, com a atualização dos valores em função da quantidade de resíduos depositados no aterro de Nova Iguaçu.

\section{Bandeirantes: projeto de gás de aterro e geração de energia}

Este aterro está situado no município de São Paulo, planejado para gerar energia elétrica por meio da queima do metano. Recebeu resíduos de 1979 a 2007, com o primeiro período de obtenção de créditos de carbono entre 2004 e 2010. Os relatórios de monitoramento (BANDEIRANTES, 2014) abrangem o período até 31 de agosto de 2012. No primeiro PDD foi usado o modelo IPCC de 1997, considerando as emissões a partir do ano de deposição. No segundo PDD, de março de 2012, empregou-se o modelo CDM Tool, com atualização das quantidades de resíduos depositados nos sete anos anteriores.

Os parâmetros originais do primeiro PDD consideraram constante de decaimento $k=0,105$ ano $^{-1}$ e potencial de geração de metano $L_{0}=$ $76,73 \mathrm{~m}^{3} \mathrm{CH}_{4} / \mathrm{t}$ resíduos. Esses valores não se mostraram coerentes com as previsões de captação de metano reportadas, recalculando-se por retroanálise $k=0,102 \mathrm{ano}^{-1} \mathrm{e} L_{0}=84,10 \mathrm{~m}^{3} \mathrm{CH}_{4} / \mathrm{t}$ resíduos, levando em conta a redução de $20 \%$ na linha de base e expectativa de eficiência de extração de $80 \%$. O segundo PDD utilizou o modelo CDM Tool.

\section{ESTRE Paulínia: projeto de recuperação de gás de aterro}

O aterro ESTRE Paulínia recebe resíduos desde 2000, com o primeiro período de obtenção de créditos entre 2006 e 2012. O primeiro PDD foi revisto para correções em janeiro de 2012, com o período de créditos modificado de 14 de setembro de 2006 a 13 de setembro de 2013. Foram feitas alterações nas quantidades anuais de resíduos depositados e nos valores dos parâmetros do modelo de previsão. A revisão foi necessária em razão do aumento substancial da produção de metano, não prevista originalmente, que se refletiria numa quantidade maior de certificados de redução de emissões do projeto (ESTRE PAULÍNIA, 2014a).

No primeiro PDD, foi utilizado o modelo IPCC (1997) com o máximo de emissões desde o ano da deposição. Já no segundo PDD, de junho de 2013, foi usado o modelo CDM Tool, com atualização em 2012 da quantidade de resíduos depositados.

No presente trabalho, os parâmetros relativos ao primeiro PDD, revisado em janeiro de 2012, foram verificados por retroanálise, confirmando-se os valores da constante de decaimento $k=0,09$ ano $^{-1}$ e do potencial de geração de metano $L_{0}=140 \mathrm{~m}^{3} \mathrm{CH}_{4} / \mathrm{t}$ resíduos, levando-se em conta redução de $20 \%$ na linha de base e expectativa de extração de $80 \%$. Constatou-se, porém, um duplo desconto relacionado ao fator de oxidação no segundo PDD (ESTRE PAULÍNIA, 2014b).

\section{Caieiras: projeto de redução de emissões de gás de aterro}

O aterro Caieiras recebe resíduos desde 2002. O primeiro período de obtenção de créditos foi de 31 de março de 2006 a 30 de março de 2013, mas o projeto começou a operar apenas em $1^{\circ}$ de fevereiro de 2007. O primeiro PDD foi revisto em janeiro de 2013 para correção das quantidades anuais de resíduos depositadas. Os relatórios de monitoramento abrangem o período de $1 .^{\circ}$ de fevereiro de 2007 a 30 de setembro de 2012 (CAIEIRAS, 2014).

No primeiro PDD foi usado o modelo IPCC (1997), mas na revisão de 2013 optou-se pelo modelo IPCC (2000), tendo sido admitido em ambos os modelos o máximo de emissões desde o ano de deposição dos resíduos. No segundo PDD, de setembro de 2013, foi empregado o modelo CDM Tool. O projeto previa desconto na linha de base equivalente à quantidade de metano usada para gerar eletricidade ou $20 \%$ da quantidade captada de metano. Como não houve geração de eletricidade, prevaleceu o desconto de $20 \%$ na linha de base. Quanto à eficiência de extração, previu-se 60\% em 2006, 70\% em 2007 e 80\% de 2008 em diante. A captação de metano ocorreu somente a partir de 2007.

Na revisão do primeiro PDD, foram utilizados a constante de decaimento $k=0,08$ ano $^{-1}$ e os seguintes dados adicionais para estimativa do potencial de geração: fração de papel, papelão e têxteis (22\%); fração de restos de alimentos e resíduos orgânicos (43\%); fração de madeiras (2\%); $M C F(1 \%) ; D O C_{\mathrm{f}}(0,77 \%) ; F(50 \%)$, correspondendo ao potencial de geração $L_{0}=116,02 \mathrm{~m}^{3} \mathrm{CH}_{4} / \mathrm{t}$ resíduos.

\section{Resumo dos parâmetros dos modelos Intergovernmental Panel on Climate Change, das quantidades de resíduos e de metano captadas nos aterros estudados}

Para os primeiros PDDs, os valores da constante de decaimento $k \mathrm{e}$ do potencial de geração de metano $L_{0}$ estão apresentados na Tabela 1 , 
juntamente com as correspondentes sugestões das diretrizes IPCC (2006) para resíduos em massa, que dependem dos parâmetros utilizados nos segundos PDDs. A composição dos resíduos de cada aterro é indicada na Tabela 2; todos têm a sua própria composição, exceto o aterro Caieiras, para o qual foram adotados os valores padrão para a América do Sul do IPCC (2006). A Tabela 3 exibe as quantidades de resíduos depositadas, as eficiências de extração projetadas nos primeiros PDDs e as quantidades de metano captadas em cada aterro.

\section{METODOLOGIA}

\section{Eficiência do modelo de previsão}

Para avaliar a capacidade de previsão de um modelo, é preciso comparar seus resultados com os valores observados. Conforme Oonk (2010), a quantidade de geração de metano pode ser obtida da Equação 15, se conhecidas a eficiência da extração $\left(\eta_{\text {extração }}\right)$ e a quantidade de metano captado:

$\eta_{\text {extração }}=$ metano captado/geração de metano

A abordagem adotada no presente trabalho foi determinar a eficiência do modelo de previsão $\left(\eta_{\text {modelo }}\right)$ definida como a razão entre a quantidade de metano captado e a estimativa de emissão prevista pelo modelo de primeira ordem, de acordo com a Equação 16.

$\eta_{\text {modelo i }}=$ metano captado/previsão de geração de metano modelo $i$

Com as quantidades anuais de metano captadas (Tabela 3), disponíveis nos relatórios de monitoramento, e as estimativas de geração

Tabela 1 - Constante de decaimento e potencial de geração de metano nos primeiros Project Design Documents.

\begin{tabular}{|c|c|c|c|c|c|c|c|}
\hline \multirow{3}{*}{ Aterro } & \multicolumn{7}{|c|}{ PDD-1 } \\
\hline & \multicolumn{3}{|c|}{$\mathrm{k}\left(\mathrm{ano}^{-1}\right)$} & \multicolumn{3}{|c|}{$\mathrm{L}_{0}\left(\mathrm{~m}^{3} \mathrm{CH}_{4} / \mathrm{t}\right.$ resíduo $)$} & \multirow{2}{*}{ Modelo } \\
\hline & PDD & Retroanálise & IPCC 2006 & PDD & Retroanálise & IPCC 2006 & \\
\hline CTR Nova Iguaçu & 0,100 & 0,100 & 0,17 & 65,67 & 117,14 & 58,37 & IPCC 1997 \\
\hline Bandeirantes & 0,105 & 0,102 & 0,17 & 76,73 & 84,10 & 73,01 & IPCC 1997 \\
\hline ESTRE Paulínia* & 0,09 & 0,090 & 0,17 & 140,00 & 140,00 & 68,71 & IPCC 1997 \\
\hline Caieiras* & 0,08 & 0,080 & 0,09 & 116,02 & 115,78 & 75,43 & IPCC 2000 \\
\hline
\end{tabular}

PDD: Project Design Document; IPCC: Intergovernmental Panel on Climate Change. *conforme PDD-1 revisado.

Tabela 2 - Frações de resíduos no segundo Project Design Document.

\begin{tabular}{|c|c|c|c|c|c|}
\hline \multirow[b]{2}{*}{ Aterro } & \multicolumn{5}{|c|}{ PDD-2 } \\
\hline & $\begin{array}{c}\text { Madeiras e produtos de } \\
\text { madeira }\end{array}$ & $\begin{array}{l}\text { Celulose, papel e } \\
\text { papelão }\end{array}$ & $\begin{array}{l}\text { Alimentos, } \\
\text { restos alimentares, } \\
\text { bebidas e tabaco }\end{array}$ & Têxteis & $\begin{array}{l}\text { Restos de jardins, } \\
\text { quintais e parques }\end{array}$ \\
\hline CTR Nova Iguaçu & $0,8 \%$ & $18,0 \%$ & $48,7 \%$ & $3,9 \%$ & ०\% \\
\hline Bandeirantes & $0,7 \%$ & $12,3 \%$ & $60,6 \%$ & $3,1 \%$ & $3,2 \%$ \\
\hline ESTRE Paulínia & $1,2 \%$ & $17,4 \%$ & $41,6 \%$ & $4,3 \%$ & O\% \\
\hline Caieiras & $4,7 \%$ & $17,1 \%$ & $44,9 \%$ & $2,6 \%$ & O\% \\
\hline
\end{tabular}

PDD: Project Design Document.

Tabela 3 - Resíduos depositados, eficiência de extração projetada nos primeiros Project Design Documents e quantidade de metano captada conforme relatórios de monitoramento.

\begin{tabular}{|c|c|c|c|c|c|c|}
\hline Aterro & Início & $\begin{array}{l}\text { Resíduos } \\
\text { depositados } \\
\text { até } 2012 \text { (t) }\end{array}$ & $\begin{array}{c}\text { Eficiência de } \\
\text { extração projetada } \\
\text { no PDD }\end{array}$ & $\begin{array}{l}\text { Número de } \\
\text { relatórios de } \\
\text { monitoramento }\end{array}$ & $\begin{array}{c}\text { Período } \\
\text { monitorado }\end{array}$ & $\begin{array}{c}\text { Metano } \\
\text { captado (t) }\end{array}$ \\
\hline CTR Nova Iguaçu & 2003 & 7.780 .010 & $85 \%$ & 6 & 6 anos & 26.292 \\
\hline Bandeirantes & 1979 & 37.226 .873 & $80 \%$ & 18 & 9 anos & 232.075 \\
\hline ESTRE Paulínia & 2000 & 13.512 .504 & $80 \%$ & 14 & 7 anos & 123.460 \\
\hline
\end{tabular}

*a eficiência de extração no aterro Caieiras foi estimada em 70\% no ano 2007. 
previstas em cada modelo, as eficiências dos modelos podem ser calculadas para cada ano dos períodos monitorados.

\section{Apresentação das eficiências dos diferentes modelos}

Na Figura 3, o primeiro resultado de eficiência refere-se ao modelo utilizado no primeiro PDD de cada projeto: o modelo IPCC (1997) para os aterros CTR Nova Iguaçu, Bandeirantes e ESTRE Paulínia; e o modelo IPCC (2000) para o aterro Caieiras. Em ambos os modelos, a constante de decaimento $k$ e o potencial de geração de metano $L_{0}$ correspondem aos valores obtidos por retroanálise, indicados na Tabela 1.

O segundo resultado que aparece na figura foi determinado com o modelo IPCC (2000), incluindo o novo fator de correção $A$ ' (Equação 8) e admitindo atraso de seis meses para o início do processo de digestão anaeróbia.

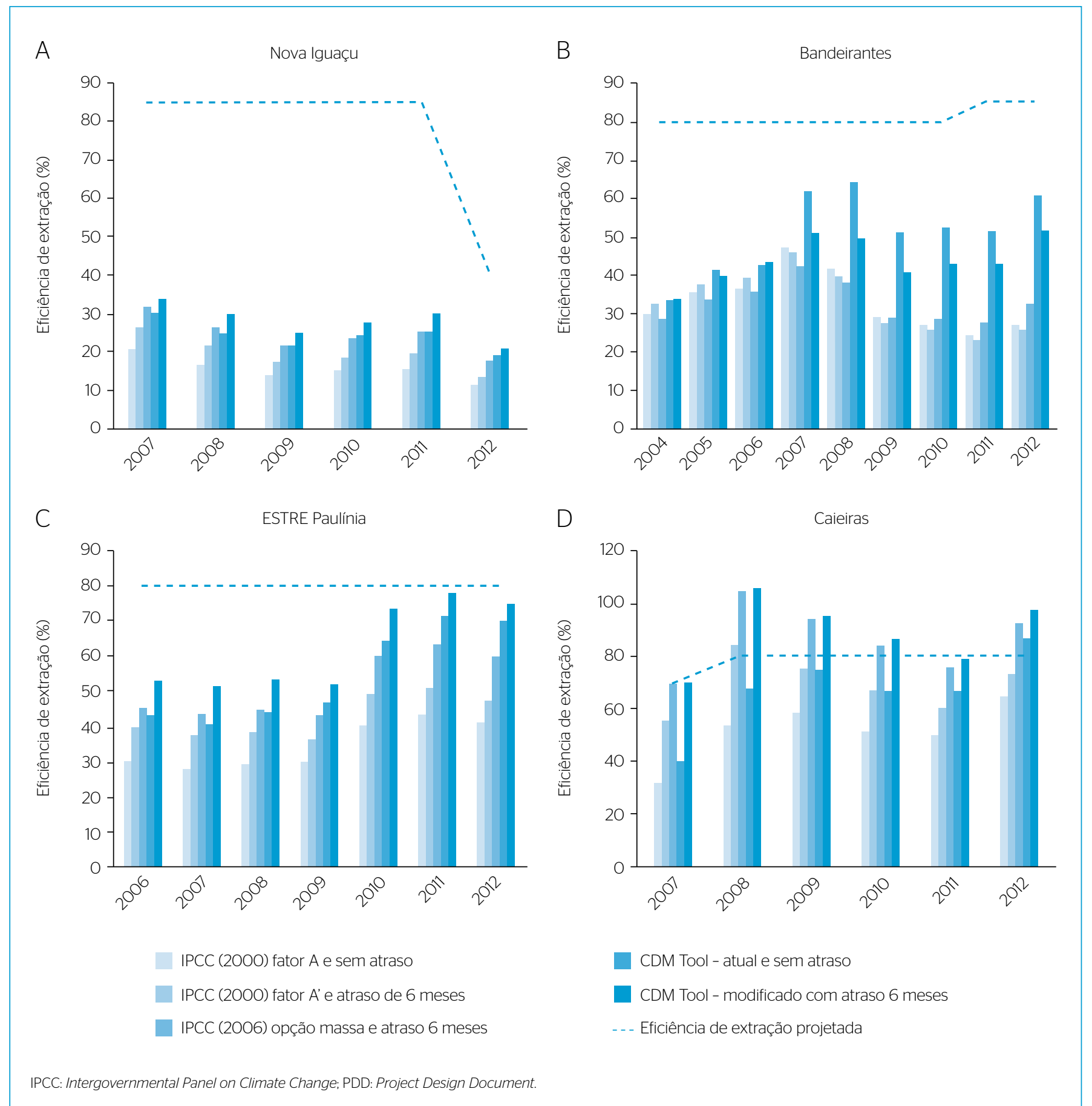

Figura 3 - Eficiências previstas por modelos Intergovernmental Panel on Climate Change e eficiência de extração projetada no Project Design Document do aterro. 
O terceiro resultado verificado foi o IPCC (2006) usando a opção massa e atraso de seis meses na decomposição dos resíduos, com constante de decaimento estabelecida de acordo com as condições de clima/umidade de cada localidade (Caieiras na condição temperado-úmido e os demais aterros na condição tropical-úmido) e com potencial de geração de metano dependente das frações de resíduos do segundo PDD de cada projeto (Tabela 2).

O quarto resultado foi determinado com o modelo CDM Tool, sem considerar o atraso de seis meses.

O quinto valor de eficiência foi obtido com o modelo CDM Tool Modificado, admitindo atraso de seis meses na decomposição dos resíduos e o novo fator de correção $A^{\prime}$ (Equação 8). Como nos primeiros PDDs, que utilizaram os modelos IPCC (1997; 2000), não existia um fator de incerteza, nos resultados apresentados para os modelos CDM Tool e CDM Tool Modificado foi então admitido um fator de incerteza $\varphi=1$. Diferentes valores do fator de incerteza podem ser aplicados posteriormente nas estimativas de emissão, com base nas Equações 11 e 14. Até a data de 25 de novembro de 2011, a ferramenta CDM Tool incorporava o fator de incerteza $\varphi=0,90$, sendo atualmente considerado $\varphi=0,75$. Observa-se que, para fator de incerteza $\varphi<1$, as estimativas de emissão diminuem, aumentando as eficiências dos modelos de previsão.
A Figura 4 apresenta a série de deposição dos resíduos entre os anos 2003 e 2012 e compara as quantidades de metano captadas, conforme relatórios de monitoramento dos aterros, com aquelas previstas pelos modelos CDM Tool e CDM Tool Modificado. As quantidades anuais de resíduos informadas nos segundos PDDs (Figura 4) foram consideradas nos modelos de previsão utilizados nos primeiros PDDs (Figura 3), para eliminar essa importante fonte de incerteza nos resultados obtidos.

\section{RESULTADOS E DISCUSSÃO}

A Figura 3 mostra eficiências de extração comparativamente mais baixas (ou previsões de emissão comparativamente mais altas) nos primeiros PDDs dos aterros de Nova Iguaçu, ESTRE Paulínia e Caieiras. Tal fato pode ser explicado pelas deficiências inerentes dos modelos empregados (IPCC 1997; 2000), como também por conta da utilização de valores elevados do potencial de geração de metano $L_{0}$, superiores aos valores padrão recomendados pelas diretrizes IPCC (2006), conforme Tabela 1.

Exceto para o aterro Bandeirantes, a consideração do segundo modelo IPCC (2000), com novo fator de correção $A^{\prime}$ e atraso de seis meses, leva a estimativas de eficiência mais altas, e a aplicação do terceiro modelo, baseado nas diretrizes IPCC (2006) na opção massa, resulta em eficiências ainda superiores.

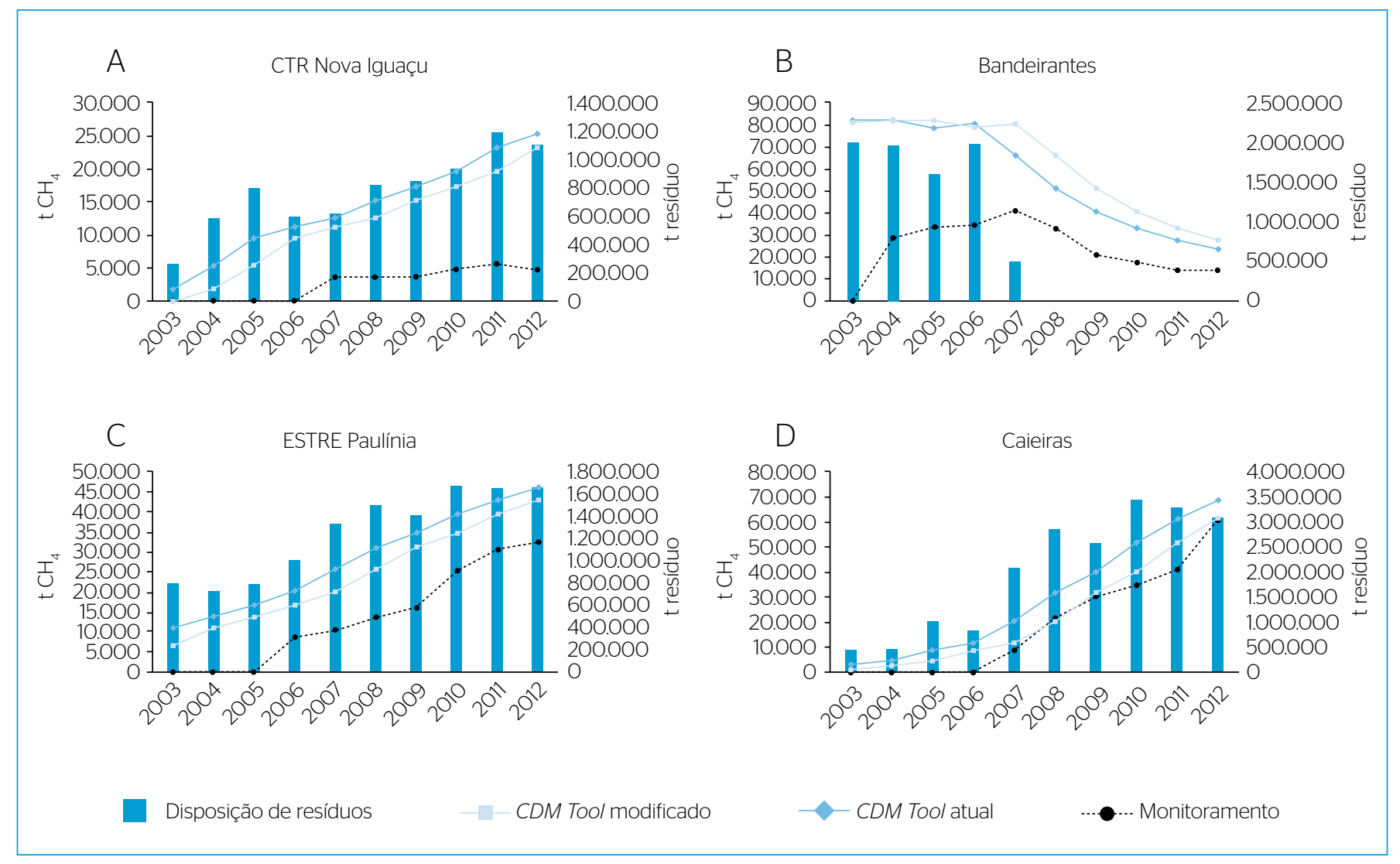

Figura 4 - Previsão de emissão de metano considerando modelos CDM Tool e CDM Tool Modificado, quantidade de metano captado de acordo com monitoramento e de rejeitos depositados anualmente. 
No segundo PDD foi empregada a abordagem das frações dos resíduos, e o modelo CDM Tool já indica melhorias de eficiência dessa ferramenta em relação aos modelos dos primeiros PDDs (IPCC 1997; 2000) e suas correções, exceto para o aterro Caieiras. O modelo CDM Tool também produz estimativas mais baixas de emissão (ou maiores eficiências) do que o modelo IPCC (2006) nos casos dos aterros Bandeirantes e ESTRE Paulínia, e mantém praticamente os mesmos valores para o aterro de Nova Iguaçu.

Quando o modelo CDM Tool é substituído pelo modelo CDM Tool Modificado, observa-se na Figura 4 que ocorre o decréscimo na previsão de captação de metano (ou aumento da eficiência do modelo) para todos os aterros, exceto para o Bandeirantes. No caso desse aterro, os resultados das previsões empregando os dois modelos são bastante próximos até o ano 2006. A partir de 2007, com o encerramento do recebimento dos resíduos no aterro, as estimativas obtidas com o modelo CDM Tool Modificado são superiores ao modelo CDM Tool. No modelo CDM Tool Modificado as emissões não são calculadas no próprio ano da deposição dos resíduos, admitindo atraso de seis meses para o início do processo de digestão anaeróbia, recebendo mais influência do histórico da deposição dos resíduos no aterro. Em outras palavras, as emissões de 2007, por exemplo, foram calculadas com base na quantidade de resíduos depositada em 2006, o que explica o maior distanciamento entre ambas as previsões a partir de 2007. Também se verifica, na Figura 4C, tendência de diminuição da geração de biogás após o encerramento do aterro, com a curva de metano captado tendendo à estabilização ao longo dos anos, fato também constatado por Silva, Freitas e Candiani (2013).

Nova Iguaçu tem os resultados mais baixos, se comparados com a eficiência de extração projetada no primeiro PDD, de 85\% (Figura 3A), e reduzida para $40 \%$ no segundo PDD, em 2011. De acordo com o segundo PDD, o sistema de extração não cobria todas as áreas que recebiam resíduos, e foi utilizada a fração de metano no biogás de $39,74 \%$, estimada com base em medições anteriores. Essa fração de metano pode ser uma indicação de que a pressão negativa empregada para a extração do gás foi excessiva, levando à diluição do biogás com o ar exterior e diminuindo a proporção de $\mathrm{CH}_{4}$ para $\mathrm{CO}_{2}$ (BINGEMER \& CRUTZEN, 1987; SCHARFF \& JACOBS, 2006). Há outras possíveis razões que poderiam explicar a baixa eficiência de extração, como problemas com a operação do aterro e perdas com emissões fugitivas e oxidação pela camada de cobertura. Deve ser também lembrado que a CTR Nova Iguaçu pertence ao primeiro conjunto de projetos de aterro que obtiveram registro MDL no mundo e que a pouca experiência brasileira na concepção e execução desse tipo de projeto pode ter contribuído para a superestimação da captação de metano no primeiro PDD.

Por outro lado, o aterro Caieiras, que serve a cidade de São Paulo, apresenta resultados elevados de eficiência. Nesse projeto, foram adotadas as frações de resíduo padrão do IPCC (2006), diferentemente do vizinho aterro Bandeirantes, que também serve São Paulo. A fração relativa a alimentos - a que mais rápido degrada - foi de 44,9\% em Caieiras e de $60,6 \%$ em Bandeirantes. Outra diferença observada é que o aterro Caieiras foi tido como situado em região de clima temperado-úmido, enquanto Bandeirantes como de clima tropical-úmido. A razão é que os aterros estão em municípios diferentes, mas em lados opostos do limite estabelecido pelo IPCC para a definição das constantes de decaimento. Se forem usados os mesmos parâmetros de Bandeirantes em Caieiras, a eficiência máxima decresce de 106 para 64\%, haja vista o modelo CDM Tool Modificado, o que parece ser uma estimativa mais razoável.

No caso do aterro Bandeirantes, a diferença entre as extrações projetada e monitorada deveu-se principalmente à má qualidade da camada de cobertura, que permitiu a intrusão do ar e o escape de biogás pela superfície do aterro. De acordo com Terraza, Willumsen e Guimarães (2007), testes executados para medir esse escape indicaram o valor médio de $0,050 \mathrm{Nm}^{3} \cdot \mathrm{h}^{-1} \cdot \mathrm{m}^{-2}$. Considerando a área total de onde o biogás era extraído, concluiu-se que uma quantidade entre $9.400 \mathrm{e}$ $24.000 \mathrm{Nm}^{3} \cdot \mathrm{h}^{-1}$ de biogás $\left(50 \% \mathrm{CH}_{4}\right)$ escapava pela superfície do aterro.

$\mathrm{Na}$ Tabela 4 os resultados são apresentados de outra forma. Foram calculadas as diferenças percentuais entre as estimativas de geração de metano consideradas nos PDDs dos projetos e as estimativas recalculadas com as correções nos respectivos modelos, para cada um dos dois períodos de obtenção de créditos de sete anos. Para o primeiro período, os modelos utilizados (IPCC 1997; 2000) levaram à superestimação significativa na captação de metano de 23 a 30\%, em três aterros em operação (CTR Nova Iguaçu, ESTRE Paulínia e Caieiras), em relação aos valores determinados com o modelo sem erros intrínsecos (IPCC 2000) (fator $A$ ' com atraso de seis meses). Já para o segundo período de créditos, o modelo empregado (CDM Tool) levou à subestimação de $13 \%$ em um aterro desativado (Bandeirantes), em relação às quantidades determinadas com o modelo sem erros intrínsecos (CDM Tool Modificado).

\section{CONCLUSÃO}

Projetos MDL, além da queda vertiginosa nos preços dos créditos de carbono, enfrentam dificuldades na previsão de captação de metano. Ainda há incertezas no estabelecimento de valores para as constantes de decaimento e potencial de geração para cada tipo de resíduo, mas as

Tabela 4 - Diferenças relativas entre emissões calculadas com modelos usados nos Project Design Documents originais e as determinadas com modelos sem os erros intrínsecos.

\begin{tabular}{l|c|c} 
Aterro & $\begin{array}{c}\text { Primeiro período } \\
\text { de créditos } \\
\text { PDD }\end{array}$ & $\begin{array}{c}\text { Segundo período } \\
\text { de créditos } \\
\text { PDD }\end{array}$ \\
\hline CTR Nova Iguaçu & $30 \%$ & $03 \%$ \\
\hline Bandeirantes & $01 \%$ & $-13 \%$ \\
\hline ESTRE Paulínia & $23 \%$ & $-08 \%$ \\
\hline Caieiras & $28 \%$ & $07 \%$ \\
\hline
\end{tabular}

PDD: Project Design Document. 
discrepâncias entre a previsão de geração e a captação efetiva de biogás em aterros sanitários podem, em parte, ser também explicadas pelo uso de modelos de previsão de primeira ordem inadequados. O próprio modelo corrente (CDM Tool) para aterros sanitários no âmbito de projetos MDL apresenta erros intrínsecos, conforme discutido.

Neste estudo comparativo entre as eficiências dos modelos de previsão IPCC, foram avaliados quatro aterros brasileiros que obtiveram um segundo período de créditos de sete anos, o que permitiu eliminar uma grande incerteza representada pela quantidade de resíduos efetivamente depositada no primeiro período.

Os modelos empregados nos primeiros PDDs (IPCC 1997; 2000) tendem a produzir, nos aterros em operação, significativas superestimações da quantidade de metano gerada em relação aos modelos IPCC sem erros intrínsecos: 30\% no CTR Nova Iguaçu, 23\% em ESTRE Paulínia, 28\% em Caieiras e 1\% em Bandeirantes, conforme Tabela 4. No modelo utilizado no segundo PDD (CDM Tool), as discrepâncias são menores em relação às previsões obtidas com o modelo CDM Tool Modificado, sem erros intrínsecos. Destaca-se, porém, a subestimação de $13 \%$ ocorrida no caso do aterro Bandeirantes, que encerrou suas atividades em 2007. O cálculo das emissões com a ferramenta CDM Tool Modificado é feita com atraso de seis meses para o início do processo de digestão anaeróbia, o que afeta os resultados quando há um súbito decréscimo na quantidade de resíduos depositada anualmente (Figura 4).

\section{REFERÊNCIAS}

AMINI, H.R.; REINHARDT, D.R.; NISKANEN, A. (2013) Comparison of first-order-decay modeled and actual field measured municipal solid waste landfill methane data. Waste Management, v. 33, n. 12, p. $2720-2728$

BANDEIRANTES. (2014) Bandeirantes Landfill Gas to Energy Project. Disponível em: <http://cdm.UNFCCC.int/Projects/DB/DNVCUK1134130255.56/view>. Acesso em: 10 set. 2014.

BARLAZ, M.A.; GREEN, R.B.; CHANTON, J.P.; GOLDSMITH, C.D.; HATER, G.R. (2004) Evaluation of a biologically active cover for mitigation of landfill gas emissions. Environmental Science and Technology, v. 38, n. 18, p. 4891-4899.

BINGEMER, H.G. \& CRUTZEN, P.J. (1987) The production of methane from solid wastes. Journal of Geophysical Research, v. 92, n. D2, p. 2181-2187.

CAIEIRAS. (2014) Caieiras Landfill Gas Emission Reduction. Disponível em: <http://cdm.UNFCCC.int/Projects/DB/DNV-CUK1134509951.62/ view>. Acesso em: 10 set. 2014

CASSINI, S.T.; BREDA, M.Z.; NASCIMENTO, T.B. (2013) Anexo B Dedução da fórmula do IPCC (2000) para estimar as emissões de $\mathrm{CH}_{4}$ pelos resíduos sólidos. In: Companhia Ambiental do Estado de São Paulo - CETESB. Emissões do setor de resíduos sólidos e efluentes líquidos, 1990 a 2008: Relatório de Referência. São Paulo: CETESB.

ESTRE PAULÍNIA. (2014a) ESTRE'S Paulinia Landfill Gas Project. Disponível em: <http://cdm.UNFCCC.int/Projects/DB/DNV-CUK1134989999.25/ view>. Acesso em: 10 set. 2014

ESTRE PAULÍNIA. (2014b) PDD2_ESTRE_Paulinia_CER sheet_165. XIsx, aba baseline emissions, linha 320, coluna R e seguintes. Disponível em: <http://cdm.unfccc.int/UserManagement/
FileStorage/ESKNVAIXRUWPJGO4LZOF1H8TYDQB36>. Acesso em: 10 set. 2014

INTERGOVERNMENTAL PANEL ON CLIMATE CHANGE - IPCC. (1997) Revised 1996 IPCC Guidelines for National Greenhouse Gas Inventories: volumes 1, 2 and 3. Paris: IPCC/OECD/IEA.

INTERGOVERNMENTAL PANEL ON CLIMATE CHANGE - IPCC. (2000) Good Practice Guidance and Uncertainty Management in National Greenhouse Gas Inventories. Hayama: IPCC/OECD/IEA/IGES.

INTERGOVERNMENTAL PANEL ON CLIMATE CHANGE - IPCC. (2006) 2006 IPCC Guidelines for National Greenhouse Gas Inventories. Japão: IGES.

NOVA IGUAÇU. (2014) Brazil NovaGerar Landfill Gas to Energy Project. Disponível em: <http://cdm.UNFCCC.int/Projects/DB/DNVCUK1095236970.6/view>. Acesso em: 10 set. 2014

OONK, H. (2010) Literature review: methane from landfills. Methods to quantify generation, oxidation and emission. Assendelft: Sustainable Landfill Foundation.

SANTOS, M.M.O. (2014) Geração de biogás em aterros sanitários: uma análise sobre os modelos de previsão aplicados a projetos do Mecanismo de Desenvolvimento Limpo. Dissertação (Mestrado Profissional em Engenharia Urbana e Ambiental) - Pontifícia Universidade Católica do Rio de Janeiro, Rio de Janeiro.

SCHARFF, H. \& JACOBS, J. (2006) Applying guidance for methane emission estimation for landfills. Waste Management, v. 26, n. 4 , p. $417-429$.

SILVA, T.N.; FREITAS, F.S.N.; CANDIANI, G. (2013) Avaliação das emissões superficiais do gás de aterros sanitários de grande porte. Revista de Engenharia Sanitária e Ambiental, v. 18, n. 2, p. 95-104. 
SPOKAS, K.; BOGNER, J.; CHANTON, J.P.; MORCET, M.; ARAN, C.; GRAFF, C.; MOREAU-LE GOLUAN, Y.; HEBE, I. (2006) Methane mass balance at three landfill sites: what is the efficiency of capture by gas collection systems? Waste Management, v. 26, n. 5 , p. 516-525.

TERRAZA, H.; WILLUMSEN, H.; GUIMARÃES, H. (2007) Landfill gas capture: design vs. actual performance and the future for CDM projects. Washington: The World Bank. 19 p.

THOMPSON, S.; SAWYER, J.; BONAM, R.; VALDIVIA, J.E. (2009) Building a better methane generation model: validating models with methane recovery rates from 35 Canadian landfills. Waste Management, v. 29, n. 7, p. 2085-2091.

UNITED NATIONS FRAMEWORK CONVENTION ON CLIMATE CHANGE - UNFCCC. (2011) Methodological Tool: emissions from solid waste disposal sites. Disponível em: <http://cdm.UNFCCC. int/methodologies/PAmethodologies/tools/am-tool-04-v6.01.pdf>. Acesso em: 10 set. 2014.

UNITED STATES ENVIRONMENTAL PROTECTION AGENCY USEPA. (2005) Landfill Gas Emissions Model (LandGEM) version 3.02 User's Guide, EPA-600/R-05/47. United States: USEPA.

VIANA, T.A.P. (2011) Análise das estimativas de emissão de metano por aterros sanitários em projetos MDL no Brasil. Dissertação (Mestrado em Engenharia Sanitária) - Universidade do Estado do Rio de Janeiro, Rio de Janeiro.

WORLD BANK (2004) Energy Sector Management Assistance Programme. Handbook for the preparation of landfill gas to energy projects in Latin America and the Caribbean. Canadá: ConestogaRovers \& Associates. 